\title{
High prevalence of dyslipidaemia subtypes and their associated personal and clinical attributes in Malaysian adults: the REDISCOVER study
}

Mohamed-Syarif Mohamed-Yassin ${ }^{1}$, Noorhida Baharudin ${ }^{1}$, Aqil Mohammad Daher ${ }^{2}$, Najmin Abu Bakar ${ }^{3}$, Anis Safura Ramli, ${ }^{1,4}$, Suraya Abdul-Razak ${ }^{1,4}$, Nor-Ashikin Mohamed Noor Khan ${ }^{5}$, Mariam Mohamad ${ }^{6}$ and Khalid Yusoff ${ }^{7,8^{*}}$ (D)

\begin{abstract}
Background: Dyslipidaemia refers to lipid abnormalities consisting of either one or any combination of the following: elevated total cholesterol (TC), elevated low-density lipoprotein cholesterol (LDL-C), elevated triglycerides (TG), and low high-density lipoprotein cholesterol (HDL-c). The prevalence of hypercholesterolaemia is steadily increasing in Malaysia. However, data on the prevalence of dyslipidaemia subtypes among Malaysians are lacking. This is important as it may have implications for preventive and management strategies for this increasing public health challenge. This study is aimed at determining the prevalence of dyslipidaemia subtypes and their associated personal and clinical attributes in Malaysians.
\end{abstract}

Methods: REDISCOVER, a prospective study, enrolled 11,288 adults where sociodemographic data, anthropometric and blood pressure measurements, fasting lipid profile and glucose, and history of diabetes, hypertension, and smoking were obtained. The cross-sectional analytic sample presented in this article comprised 10,482 participants from baseline recruitment. The data was analysed by descriptive statistics and multivariable logistic regression.

Results: The overall prevalence of elevated TC, elevated LDL-C, elevated TG, low HDL-c, and elevated non-HDL-c were 64.0\% (95\% Cl 63.0-65.0), 56.7\% (Cl 55.7-57.7), 37.4\% (Cl 36.5-38.4), 36.2\% (Cl 35.2-37.1), and 56.2\% (Cl 55.3-57.2), respectively. Overweight, obesity, and central obesity were highly prevalent and significantly associated with elevated TC and all dyslipidaemia subtypes. Older age was associated with elevated TC, elevated LDL-c and elevated non-HDLc. Hypertension was associated with elevated TC, elevated TG, and elevated non-HDL-C, while diabetes was associated with elevated TG and low HDL-c.

Conclusions: Elevated TC and all dyslipidaemia subtypes are highly prevalent in Malaysia where increased body mass seems the main driver. Differences in the prevalence and associated personal and clinical attributes may facilitate specific preventive and management strategies.

Keywords: Dyslipidaemia, LDL-C, HDL-C, Non-HDL-c, Triglycerides, Hypercholesterolaemia, Malaysia, Prevalence

*Correspondence: khalid@ucsiuniversity.edu.my

${ }^{7}$ Faculty of Medicine and Health Sciences, UCSI University, No.1, Jalan Menara Gading, UCSI Heights, Taman Connaught, 56000 Cheras, Kuala Lumpur, Malaysia

Full list of author information is available at the end of the article

\section{Background}

Dyslipidaemia refers to lipid abnormalities consisting of either one or any combination of the following: elevated total cholesterol (TC), elevated low-density 
lipoprotein cholesterol (LDL-c), elevated triglycerides (TG), and low high-density lipoprotein cholesterol (HDL-c) [1]. Whilst TC, and particularly LDL-c are established as the most important cardiovascular risk factors, recent evidence suggests that non-high density lipoprotein cholesterol (non-HDL-c) is a more powerful cardiovascular (CV) risk predictor than LDL-c alone [2-4]. Non-HDL-c encompasses the total amount of atherogenic lipoproteins [very low-density lipoprotein (VLDL), VLDL remnants, intermediatedensity lipoprotein (IDL), LDL-c and lipoprotein(a)] [1]. TG has also been considered to be an important $\mathrm{CV}$ risk factor either independently, or as a component of atherogenic dyslipidaemia, along with low HDL-c and elevated levels of small dense LDL particles $[5,6]$.

The World Health Organization (WHO) reported that the prevalence of hypercholesterolaemia were the highest in Europe (53.7\%) and America (47.7\%), while South East Asia (30.3\%) and Africa (23.1\%) had much lower prevalence [7]. However, Lin et al, reported marked prevalence differences between different Asia Pacific countries, ranging from 9\% in Indonesia to $46.9 \%$ in the Philippines [8]. For high LDL-c, high TG, and low HDL-c, the prevalence ranges from 7.8 to $47.2 \%, 13.9$ to $38.6 \%$, and 10.1 to $71.3 \%$, respectively [8].

In Malaysia, the National Health and Morbidity Surveys (NHMS) reported the increasing prevalence of hypercholesterolaemia from $20.7 \%$ in 2006 [9], 35.1\% in 2011 [10] and $47.7 \%$ in 2015 [11]. Small scale studies have assessed the prevalence of dyslipidaemia in specific populations such as rural Malays [12] and male factory workers [13]. However, to the best of our knowledge, there has been no report on the prevalence of non-HDL-c in Malaysians. The current Malaysian dyslipidaemia management guideline recommends that LDL-c should be the primary treatment target [1]. It also suggests that non-HDL-c can be used as a secondary target for individuals with combined hyperlipidaemias, diabetes, metabolic syndrome, or chronic kidney disease [1]. Therefore, it is pertinent to establish the prevalence of all dyslipidaemia subtypes including high non-HDL-c in order to achieve individualised patient targets as recommended by the national guideline.

We report the prevalence of elevated total cholesterol and each dyslipidaemia subtype including high non-HDL-c in Malaysian adults aged $\geq 30$ years from the REDISCOVER study. We also examine the relationship between these dyslipidaemia subtypes with personal and clinical attributes in this population.

\section{Methods}

\section{Study design and population}

The REDISCOVER (Responding to Increasing Cardiovascular Disease Prevalence) study is an ongoing prospective study involving Malaysians aged 30 years and above, involving 22 rural and 18 urban communities from five states across Malaysia. The states involved are Selangor, Negri Sembilan, Pahang, Kelantan, and Sabah.

\section{Sampling methods: state and site selection}

The five states were selected to ensure satisfactory representation of the major ethnic groups in Malaysia. In Peninsular Malaysia, the main ethnic groups are the Malays, Chinese and Indians. In Sabah (East Malaysia), the major ethnic groups are the Kadazan-Dusun, Bajau, and Murut. For this study, these groups from Sabah along with several other ethnic minorities were classified as the indigenous group. The other states selected (Selangor, Negri Sembilan and Pahang) have a robust mix of Malays, Chinese and Indians. Kelantan consists of a majority Malay populace, while Sabah represents the indigenous population.

\section{Sampling methods: subjects recruitment}

A standardized method of recruitment was used. Announcements and invitation were made via local community leaders. Written invitation was issued for all household member above 30 years old to attend screening sessions at local community centres with an eight hour fast. The response rate for each site was between 60 to $70 \%$. At the screening centres, the participants were screened for eligibility and an informed consent obtained. The study was approved by the institutional ethics committee [Ethics approval number: REC/UITM/2007(10)].

\section{Study procedures}

All investigators and interviewers underwent training on the study procedures. A standard data collection form recorded the age, gender, ethnicity, educational attainment, smoking status, diabetes mellitus, hypertension, cardiovascular disease (CVD), and use of cholesterol-lowering medication. Education attainment was divided into four subgroups i.e. "no formal education", "primary", "secondary", and "tertiary". Primary education was defined as schooling from the ages of seven to 12 years old, while secondary education was schooling from the ages of 13 to 17 years old, and tertiary education attainment of college or university education. Population of $\geq 10,000$ was classified as urban 
Table 1 Sociodemographic and clinical characteristics $(\mathrm{N}=10,482)$

Sociodemographic and clinical characteristics

\begin{tabular}{|c|c|}
\hline Age (years) ( $\pm S D)$ & $52.7( \pm 11.1)$ \\
\hline \multicolumn{2}{|l|}{ Age groups (years) $(n, \%)$} \\
\hline $30-39$ & $1207(11.5)$ \\
\hline $40-49$ & $3196(30.5)$ \\
\hline $50-59$ & $3277(31.3)$ \\
\hline$\geq 60$ & $2802(26.7)$ \\
\hline \multicolumn{2}{|l|}{ Gender $(n, \%)$} \\
\hline Male & $4538(43.3)$ \\
\hline Female & $5944(56.7)$ \\
\hline \multicolumn{2}{|l|}{ Ethnicity (n,\%) } \\
\hline Malay & 7642 (72.9) \\
\hline Chinese & 1049 (10.0) \\
\hline Indian & $276(2.6)$ \\
\hline Indigenous & $1515(14.5)$ \\
\hline \multicolumn{2}{|l|}{ Education attainment ( $\left.n^{i}, \%\right)$} \\
\hline No formal education & 1499 (15.8) \\
\hline Primary school & $2589(27.3)$ \\
\hline Secondary school & $3628(38.3)$ \\
\hline Tertiary & $1762(18.6)$ \\
\hline \multicolumn{2}{|l|}{ Location (n,\%) } \\
\hline Urban & $5262(50.2)$ \\
\hline Rural & $5220(49.8)$ \\
\hline \multicolumn{2}{|l|}{ Smoking status ( $\left.n^{i \prime}, \%\right)$} \\
\hline Non-smoker & $7431(75.7)$ \\
\hline Ex-smoker & $1080(11.0)$ \\
\hline Current smoker & 1307 (13.3) \\
\hline \multicolumn{2}{|l|}{ Diabetes mellitus $(n, \%)$} \\
\hline No & $8902(84.9)$ \\
\hline Yes & $1580(15.1)$ \\
\hline \multicolumn{2}{|l|}{ Hypertension ( $n, \%)$} \\
\hline No & $5663(54.0)$ \\
\hline Yes & $4819(46.0)$ \\
\hline \multicolumn{2}{|l|}{ Self-reported cardiovascular disease (n,\%) } \\
\hline No & $10,039(95.8)$ \\
\hline Yes & $443(4.2)$ \\
\hline \multicolumn{2}{|l|}{ Body mass index $\left(\mathrm{kg} / \mathrm{m}^{2}\right)\left(\mathrm{n}^{i v}, \%\right)$} \\
\hline Underweight $(<18.5)$ & $385(3.9)$ \\
\hline Normal $(<22.9)$ & $2377(24.0)$ \\
\hline Overweight (23-27.4) & $3814(38.5)$ \\
\hline Obese (> 27.4) & $3326(33.6)$ \\
\hline \multicolumn{2}{|l|}{ Waist-hip ratio $\left(n^{\vee}, \%\right)$} \\
\hline Normal & $4303(43.7)$ \\
\hline Abdominal obesity (Male $\geq 0.90$; female $\geq 0.85$ ) & $5539(56.3)$ \\
\hline
\end{tabular}

and those with $<10,000$ as rural (Malaysian Population and Housing Census 2000) [14]. Smoking was classified as: 1 . Current smokers were those who were currently smoking or, had smoked any tobacco products within the recent five years, 2. Non-smokers were those who had never smoked, 3. Ex-smokers were those who had quit smoking for more than five years. Diabetes was defined as fasting plasma glucose at $\geq 7.0 \mathrm{mmol} / \mathrm{L}$ and/ or self-reported diabetes, and/or taking medications for diabetes in the past month. Cardiovascular diseases included ischaemic heart disease (IHD) and stroke that were reported by the participants.

Waist and hip circumferences were measured (to the nearest $0.1 \mathrm{~cm}$ ) using a non-stretchable measuring tape while the participants stood in a relaxed position with arms by their side. Blood pressure was measured using the Omron automatic digital blood pressure monitors (Omron HEM-757) after a five-minute rest. Right arm BP measurements were taken on two occasions, two minutes apart while participants were seated, and a mean of the two readings was taken as the BP for the participant.

Participants were classified based on body mass index (BMI in $\mathrm{kgm}^{-2}$ ) into: Underweight $<18.5$, normal 18.5 22.9 , overweight $23-27.4$, or obese $\geq 27.5$ [15]. Abdominal or central obesity was defined as waist-hip ratio (WHR) of $\geq 0.90$ and $\geq 0.85$, in males and females respectively [16]. Hypertension was defined as mean systolic BP of $\geq 140$ and/or mean diastolic $\mathrm{BP} \geq 90 \mathrm{mmHg}$; or selfreported hypertension; or taking antihypertensive medications in the last month.

Fasting venous blood samples were collected for serum lipid profile [total cholesterol (TC), triglycerides (TG), high-density lipoprotein cholesterol (HDL-c), low-density lipoprotein cholesterol (LDL-c)] and plasma glucose. All variables, except for LDL-c were analysed using an automated clinical chemical analyzer (Cobas Integra 400 plus, Roche Diagnostic, Basel, Switzerland). LDL-c was calculated using the Freidewald equation (for TG $\leq 4.5 \mathrm{mmol} / \mathrm{L}$ ) [1]. Non-HDL-c was calculated using the following equation TC-HDL-c (mmol/L) [1]. Dyslipidaemia is defined as either one or a combination of the following lipid levels [1]:

- $\mathrm{TC}>5.2 \mathrm{mmol} / \mathrm{L}$;

- LDL-c > $3.4 \mathrm{mmol} / \mathrm{L}$;

- $\mathrm{TG}>1.7 \mathrm{mmol} / \mathrm{L}$;

- HDL-c $<1$ in males; $<1.2 \mathrm{mmol} / \mathrm{L}$ in females;

- Non-HDL-c levels were considered high if values $>4.2 \mathrm{mmol} / \mathrm{L}[1]$. 


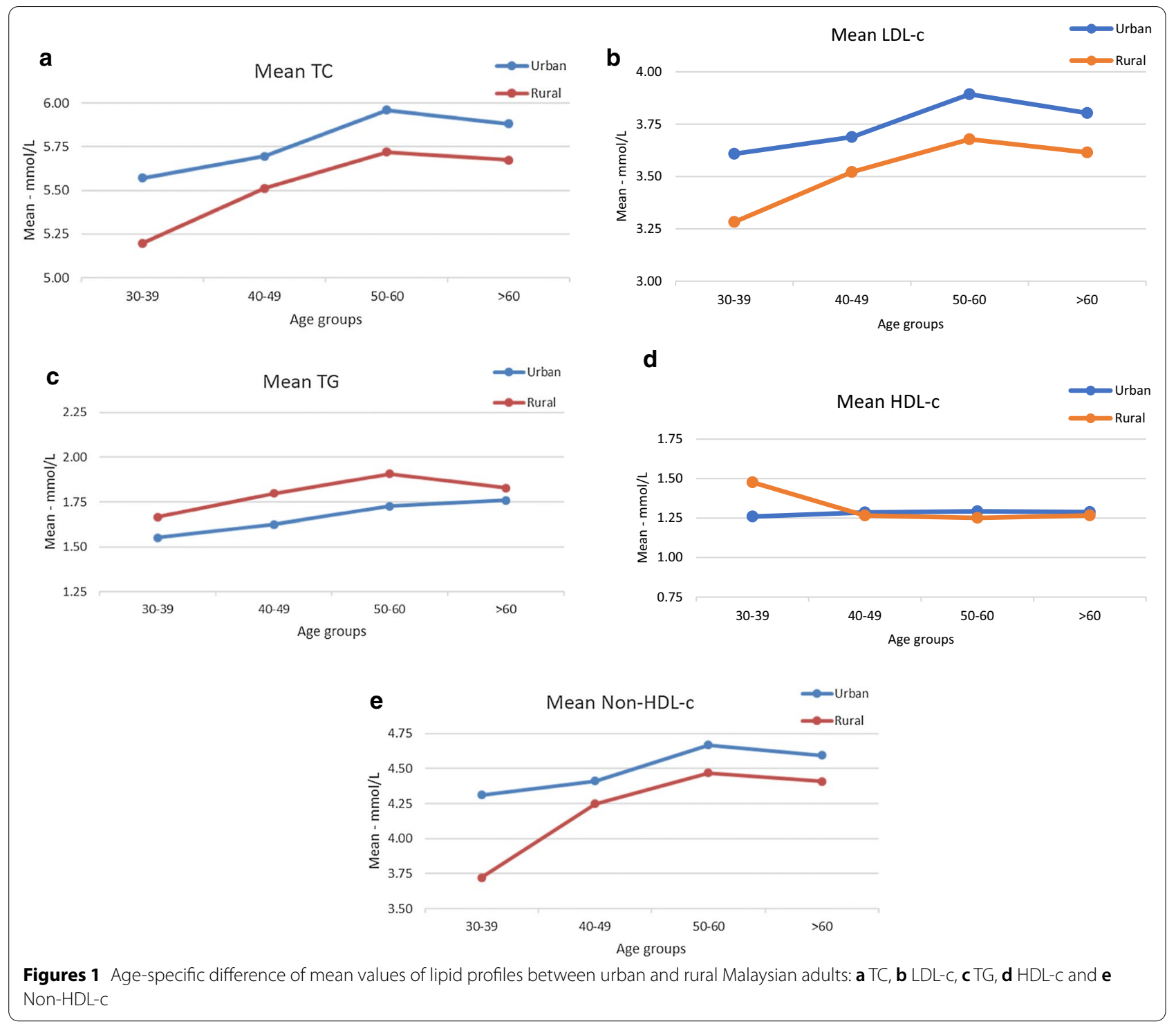

\section{Data collection}

The REDISCOVER study duration is 15 years and the baseline data were collected from 2007 to 2011. Data are being collected every three years. A total of 11,288 participants were recruited, with 806 participants who were taking cholesterol-lowering medication excluded from this analysis. The cross-sectional analytic sample presented in this article comprised 10,482 participants from baseline recruitment.

\section{Statistical analysis}

Data were entered and analyzed using STATA software version 14 (StataCorp.TX). Missing data was automatically removed during analysis via pairwise deletion where cases without specific variable data were excluded from analysis of that variable only. Categorical variables were presented using frequencies and percentage, while numerical variables were presented using mean $( \pm$ standard deviations [SD]). Chi-square test was used to compare categorical variables. Sensitivity analysis was done by comparing crude and age-adjusted prevalence. To estimate the crude and adjusted odds ratio for the factors associated with the subtypes of dyslipidaemia, simple and multiple regression models were utilized, and interactions were checked. Sample size was calculated for estimating prevalence of 0.5 with 0.03 margin of error and 95\% CI was 964 . To accommodate for design effect of 4 and non-response rate of $30 \%$, the final sample size was 3855. A $p$ value of less than 0.05 was considered statistically significant. 


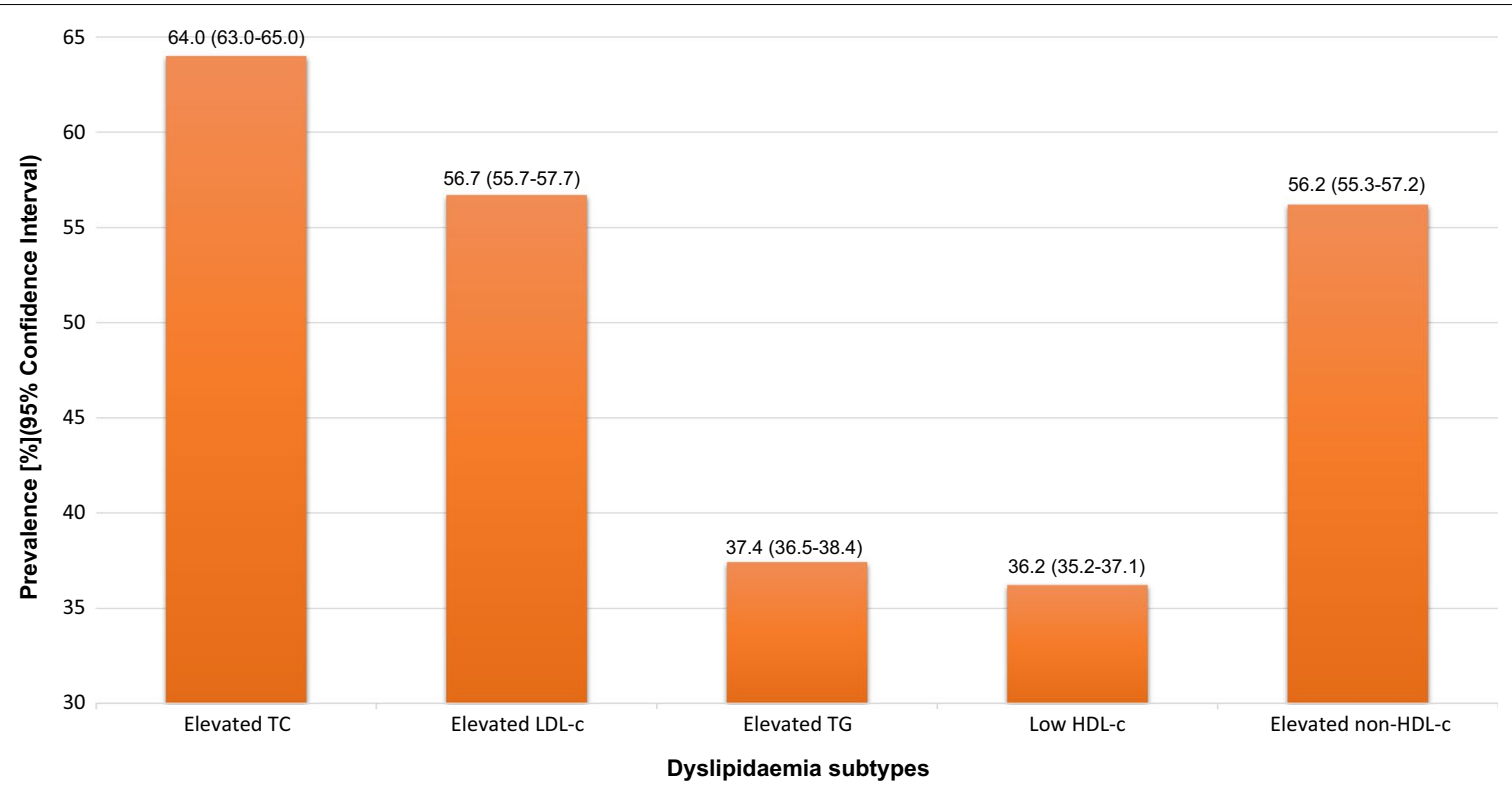

Fig. 2 Overall prevalence of elevated TC \& dyslipidaemia subtypes

\section{Results}

\section{Characteristics of the participants}

10,482 participants were included in this analysis. The sociodemographic and clinical characteristics of the participants are shown in Table 1. The mean age was $52.7( \pm 11.1)$ years. There were more females than males (56.7\% vs. $43.3 \%)$. Malays were the predominant ethnicity (72.9\%), and there was an almost equal split of urban and rural participants $(50.2 \%$ vs. $49.8 \%)$. Almost thirty nine percent of the participants were overweight while $33.6 \%$ were obese. Forty six percent of the participants had hypertension, $15.1 \%$ had diabetes and $13.3 \%$ were current smokers.

Figure 1a-e show the age-specific difference of mean values of lipid profiles between urban and rural Malaysian adults. The mean TC, LDL-c and non-HDL-c levels were consistently higher in the urban participants compared to rural participants across all age groups. In contrast, the mean TG levels were consistently higher in the rural participants compared to the urban ones across all age groups.

\section{Prevalence of dyslipidaemia subtypes}

The overall prevalence of elevated TC, elevated LDL-c, elevated TG, and low HDL-c were $64.0 \%$ (CI 63.0-65.0), 56.7\% (CI 55.7-57.7), 37.4\% (CI 36.5-38.4) and 36.2\% (CI 35.2-37.1), respectively. The overall prevalence of elevated non-HDL-c was 56.2\% (CI 55.3-57.2) (Fig. 2). There was no difference between the crude and ageadjusted prevalence.
Elevated TC and elevated LDL-c were more prevalent among Malays, urban participants, those with hypertension, obese, and centrally obese participants (Tables 2 and 3). Elevated TG was more prevalent among males (46.2\%), rural participants $(40.4 \%)$, those with diabetes (55.8\%), hypertension (44.6\%), obese (74.7\%), and centrally obese (49.0\%) (Table 4).

For low HDL-c, Indigenous participants (52\%), current smokers (45.7\%), participants with diabetes (44.1\%), hypertension (37.4\%), obesity (45.6\%), and central obesity $(43.8 \%)$ had higher prevalence compared to the others (Table 5). Elevated non-HDL-c was more prevalent in males $(60.8 \%)$, Malays $(62.5 \%)$, urban participants $(58.5 \%)$, those with diabetes $(64.5 \%)$, hypertension $(61.3 \%)$, obese $(65.6 \%)$, and centrally obese $(63.6 \%)$ (Table 6).

\section{Personal and clinical attributes associated with dyslipidaemia subtypes}

Table 7 displays a multiple logistic regression model used to ascertain the personal and clinical attributes associated with the subtypes of dyslipidaemia. Compared to the 30 to 39 age group, those in the 50 to 59 age group were associated with higher adjusted odds ratio for all subtypes of dyslipidaemia except for elevated TG, and lower adjusted odds ratio for low HDL-c. Females had increased likelihood of low HDL-c [aOR 1.14 (95\% CI 1.02-1.29)], but reduced likelihood of high TG [aOR 0.62 (95\% CI 0.55-0.70)] and high non-HDL-c [aOR 0.79 (95\% CI 0.71-0.88)], compared to males. 
Table 2 Mean TC and prevalence of elevated TC according to personal and clinical attributes

\begin{tabular}{|c|c|c|c|c|c|}
\hline & \multirow[t]{2}{*}{ Mean $( \pm S D)$} & \multirow[b]{2}{*}{$p$ value } & \multicolumn{3}{|l|}{ Elevated TC } \\
\hline & & & $\begin{array}{l}\text { No } \\
\text { n (\%) }\end{array}$ & $\begin{array}{l}\text { Yes } \\
\text { n (\%) }\end{array}$ & $p$ value \\
\hline $\begin{array}{l}\text { Age groups (years) } \\
30-39 \\
40-49 \\
50-59 \\
\geq 60\end{array}$ & $\begin{array}{l}5.39( \pm 1.15) \\
5.61( \pm 1.14) \\
5.85( \pm 1.28) \\
5.76( \pm 1.29)\end{array}$ & $<0.001^{* *}$ & $\begin{array}{l}516(45.5) \\
1177(38.5) \\
979(31.0) \\
948(35.1)\end{array}$ & $\begin{array}{l}619(54.5) \\
1880(61.5) \\
2177(69.0) \\
1751(64.9)\end{array}$ & $<0.001^{* *}$ \\
\hline $\begin{array}{l}\text { Gender } \\
\text { Male } \\
\text { Female }\end{array}$ & $\begin{array}{l}5.68( \pm 1.24) \\
5.72( \pm 1.23)\end{array}$ & 0.129 & $\begin{array}{l}1564(36.2) \\
2056(35.9)\end{array}$ & $\begin{array}{l}2753(63.8) \\
3674(64.1)\end{array}$ & 0.719 \\
\hline $\begin{array}{l}\text { Ethnicity } \\
\text { Malay } \\
\text { Chinese } \\
\text { Indian } \\
\text { Indigenous }\end{array}$ & $\begin{array}{l}5.89( \pm 1.21) \\
5.54( \pm 1.10) \\
5.51( \pm 1.12) \\
4.90( \pm 1.15)\end{array}$ & $<0.001^{* *}$ & $\begin{array}{l}2178(29.8) \\
406(40.1) \\
110(40.4) \\
926(64.0)\end{array}$ & $\begin{array}{l}5136(70.2) \\
607(59.9) \\
162(59.6) \\
522(36.1)\end{array}$ & $<0.001^{* *}$ \\
\hline $\begin{array}{l}\text { Education attainment } \\
\text { No formal education } \\
\text { Primary } \\
\text { Secondary } \\
\text { Tertiary }\end{array}$ & $\begin{array}{l}5.51( \pm 1.34) \\
5.77( \pm 1.26) \\
5.75( \pm 1.19) \\
5.72( \pm 1.11)\end{array}$ & $<0.001^{* *}$ & $\begin{array}{l}640(44.0) \\
860(34.6) \\
1181(33.9) \\
581(34.1)\end{array}$ & $\begin{array}{l}814(56.0) \\
1627(65.4) \\
2302(66.1) \\
1125(65.9)\end{array}$ & $<0.001^{* *}$ \\
\hline $\begin{array}{l}\text { Location } \\
\text { Urban } \\
\text { Rural }\end{array}$ & $\begin{array}{l}5.81( \pm 1.16) \\
5.59( \pm 1.30)\end{array}$ & $<0.001^{* *}$ & $\begin{array}{l}1597(31.7) \\
2023(40.4)\end{array}$ & $\begin{array}{l}3437(68.3) \\
2990(59.6)\end{array}$ & $<0.001^{* *}$ \\
\hline $\begin{array}{l}\text { Smoking status } \\
\text { Non-smoker } \\
\text { Previous smoker } \\
\text { Current smoker }\end{array}$ & $\begin{array}{l}5.71( \pm 1.21) \\
5.69( \pm 1.27) \\
5.71( \pm 1.27)\end{array}$ & 0.830 & $\begin{array}{l}2577(35.9) \\
366(35.0) \\
441(36.0)\end{array}$ & $\begin{array}{l}4611(64.2) \\
681(65.0) \\
784(64.0)\end{array}$ & 0.839 \\
\hline $\begin{array}{l}\text { Diabetes mellitus } \\
\text { No } \\
\text { Yes }\end{array}$ & $\begin{array}{l}5.67( \pm 1.22) \\
5.86( \pm 1.31)\end{array}$ & $<0.001^{* *}$ & $\begin{array}{l}3127(36.7) \\
493(32.1)\end{array}$ & $\begin{array}{l}5386(63.3) \\
1041(67.9)\end{array}$ & $0.001^{*}$ \\
\hline $\begin{array}{l}\text { Hypertension } \\
\text { No } \\
\text { Yes }\end{array}$ & $\begin{array}{l}5.60( \pm 1.20) \\
5.82( \pm 1.27)\end{array}$ & $<0.001^{* *}$ & $\begin{array}{l}2101(39.0) \\
1519(32.6)\end{array}$ & $\begin{array}{l}3290(61.0) \\
3137(67.4)\end{array}$ & $<0.001^{* *}$ \\
\hline $\begin{array}{l}\text { Body mass index } \\
\text { Underweight } \\
\text { Normal } \\
\text { Overweight } \\
\text { Obese }\end{array}$ & $\begin{array}{l}5.20( \pm 1.12) \\
5.47( \pm 1.21) \\
5.79( \pm 1.23) \\
5.87( \pm 1.20)\end{array}$ & $<0.001^{* *}$ & $\begin{array}{l}202(54.5) \\
1005(43.9) \\
1221(33.3) \\
962(30.0)\end{array}$ & $\begin{array}{l}169(45.6) \\
1283(56.1) \\
2451(66.8) \\
2250(70.1)\end{array}$ & $<0.001^{* *}$ \\
\hline $\begin{array}{l}\text { Waist-hip ratio } \\
\text { Normal } \\
\text { Abdominal obesity }\end{array}$ & $\begin{array}{l}5.60( \pm 1.16) \\
5.83( \pm 1.27)\end{array}$ & $<0.001^{* *}$ & $\begin{array}{l}1818(39.0) \\
1660(32.9)\end{array}$ & $\begin{array}{l}2847(61.0) \\
3379(67.1)\end{array}$ & $<0.001^{* *}$ \\
\hline
\end{tabular}

* significant at $p<0.05 ;{ }^{* *}$ significant at $p<0.001$

The Indigenous population had lower odds for elevated TC [aOR 0.27 (95\% CI 0.23-0.32)], elevated LDL-c [aOR 0.29 (95\% CI 0.25-0.34)], and elevated non-HDL-c [aOR 0.33 (95\% CI 0.28-0.39)], compared to Malays. However, they had almost 2.5 times [aOR 2.42 (95\% CI 2.08-2.81)] increased odds of having low HDL-c. Compared to urban participants, rural participants had increased likelihood of having high TG [aOR 1.39 (95\% CI 1.24-1.57)], but they were less likely to have high LDL-c [aOR 0.84 (95\% CI 0.75-0.95)].
Overweight, obesity and central obesity were significantly associated with elevated TC and all subtypes of dyslipidaemia compared to normal BMI and normal waist-hip ratios. In contrast, participants who were underweight were associated with reduced odds for elevated TC and all dyslipidaemia subtypes except for low HDL-c.

Participants with hypertension had increased adjusted OR for elevated TC, elevated TG, and elevated nonHDL-c. Participants with diabetes had increased likelihood of elevated TG [aOR 1.90 (95\% CI 1.67-2.16)] and reduced HDL-c [aOR 1.34 (95\% CI 1.18-1.53)]. 
Table 3 Mean LDL-c and prevalence of elevated LDL-c according to personal and clinical attributes

\begin{tabular}{|c|c|c|c|c|c|}
\hline & \multirow[t]{2}{*}{ Mean ( $\pm S D)$} & \multirow[b]{2}{*}{$p$ value } & \multicolumn{3}{|c|}{ Elevated LDL-c } \\
\hline & & & $\begin{array}{l}\text { No } \\
n(\%)\end{array}$ & $\begin{array}{l}\text { Yes } \\
\text { n (\%) }\end{array}$ & $p$ value \\
\hline $\begin{array}{l}\text { Age groups (years) } \\
30-39 \\
40-49 \\
50-59 \\
\geq 60\end{array}$ & $\begin{array}{l}3.45( \pm 1.01) \\
3.61( \pm 1.03) \\
3.79( \pm 1.14) \\
3.69( \pm 1.12)\end{array}$ & $<0.001^{* *}$ & $\begin{array}{l}570(50.3) \\
1368(44.8) \\
1242(39.4) \\
1169(43.3)\end{array}$ & $\begin{array}{l}564(49.7) \\
1689(55.3) \\
1910(60.6) \\
1529(56.7)\end{array}$ & $<0.001^{* *}$ \\
\hline $\begin{array}{l}\text { Gender } \\
\text { Male } \\
\text { Female }\end{array}$ & $\begin{array}{l}3.69( \pm 1.10) \\
3.66( \pm 1.09)\end{array}$ & 0.245 & $\begin{array}{l}1817(42.1) \\
2532(44.2)\end{array}$ & $\begin{array}{l}2497(57.9) \\
3195(55.8)\end{array}$ & $0.036^{*}$ \\
\hline $\begin{array}{l}\text { Ethnicity } \\
\text { Malay } \\
\text { Chinese } \\
\text { Indian } \\
\text { Indigenous }\end{array}$ & $\begin{array}{l}3.84( \pm 1.07) \\
3.42( \pm 0.98) \\
3.55( \pm 1.00) \\
3.02( \pm 1.01)\end{array}$ & $<0.001^{* *}$ & $\begin{array}{l}2696(36.9) \\
521(51.6) \\
125(46.0) \\
1007(69.5)\end{array}$ & $\begin{array}{l}4616(63.1) \\
488(48.4) \\
147(54.0) \\
441(30.5)\end{array}$ & $<0.001^{* *}$ \\
\hline $\begin{array}{l}\text { Education attainment } \\
\text { No formal education } \\
\text { Primary } \\
\text { Secondary } \\
\text { Tertiary }\end{array}$ & $\begin{array}{l}3.49( \pm 1.15) \\
3.72( \pm 1.12) \\
3.72( \pm 1.07) \\
3.70( \pm 1.00)\end{array}$ & $<0.001^{* *}$ & $\begin{array}{l}753(51.8) \\
1053(42.3) \\
1428(41.1) \\
696(40.8)\end{array}$ & $\begin{array}{l}700(48.2) \\
1434(57.7) \\
2050(58.9) \\
1010(59.2)\end{array}$ & $<0.001^{* *}$ \\
\hline $\begin{array}{l}\text { Location } \\
\text { Urban } \\
\text { Rural }\end{array}$ & $\begin{array}{l}3.77( \pm 1.04) \\
3.57( \pm 1.13)\end{array}$ & $<0.001^{* *}$ & $\begin{array}{l}1954(38.9) \\
2395(47.8)\end{array}$ & $\begin{array}{l}3076(61.2) \\
2616(52.2)\end{array}$ & $<0.001^{* *}$ \\
\hline $\begin{array}{l}\text { Smoking status } \\
\text { Non-smoker } \\
\text { Previous smoker } \\
\text { Current smoker }\end{array}$ & $\begin{array}{l}3.67( \pm 1.08) \\
3.71( \pm 1.13) \\
3.70( \pm 1.09)\end{array}$ & 0.456 & $\begin{array}{l}3131(43.6) \\
448(42.8) \\
502(41.0)\end{array}$ & $\begin{array}{l}4052(56.4) \\
599(57.2) \\
722(59.0)\end{array}$ & 0.235 \\
\hline $\begin{array}{l}\text { Diabetes mellitus } \\
\text { No } \\
\text { Yes }\end{array}$ & $\begin{array}{l}3.67( \pm 1.09) \\
3.69( \pm 1.13)\end{array}$ & 0.479 & $\begin{array}{l}3704(43.5) \\
645(42.1)\end{array}$ & $\begin{array}{l}4804(56.5) \\
888(57.9)\end{array}$ & 0.288 \\
\hline $\begin{array}{l}\text { Hypertension } \\
\text { No } \\
\text { Yes }\end{array}$ & $\begin{array}{l}3.62( \pm 1.05) \\
3.74( \pm 1.14)\end{array}$ & $<0.001^{* *}$ & $\begin{array}{l}2455(45.6) \\
1894(40.7)\end{array}$ & $\begin{array}{l}2934(54.4) \\
2758(59.3)\end{array}$ & $<0.001^{* *}$ \\
\hline $\begin{array}{l}\text { Body mass index } \\
\text { Underweight } \\
\text { Normal } \\
\text { Overweight } \\
\text { Obese }\end{array}$ & $\begin{array}{l}3.20( \pm 0.96) \\
3.45( \pm 1.06) \\
3.76( \pm 1.11) \\
3.80( \pm 1.06)\end{array}$ & $<0.001^{* *}$ & $\begin{array}{l}244(65.8) \\
1198(52.4) \\
1468(40.0) \\
1201(37.4)\end{array}$ & $\begin{array}{l}127(34.2) \\
1089(47.6) \\
2202(60.0) \\
2008(62.6)\end{array}$ & $<0.001^{* *}$ \\
\hline $\begin{array}{l}\text { Waist-hip ratio } \\
\text { Normal } \\
\text { Abdominal obesity }\end{array}$ & $\begin{array}{l}3.59( \pm 1.04) \\
3.76( \pm 1.13)\end{array}$ & $<0.001^{* *}$ & $\begin{array}{l}2061(46.6) \\
1814(39.9)\end{array}$ & $\begin{array}{l}2364(53.4) \\
2737(60.1)\end{array}$ & $<0.001^{* *}$ \\
\hline
\end{tabular}

* significant at $p<0.05 ;{ }^{* *}$ significant at $p<0.001$

\section{Discussion}

The REDISCOVER Study found that almost two-thirds of participants have elevated TC. In comparison with the National Health and Morbidity Survey 2011 (NHMS 2011) which included participants above 18 years old, the prevalence of hypercholesterolaemia in our study was nearly twice as high (64.0\% vs. $35.1 \%)$ [10]. The national prevalence of hypercholesterolaemia has shown an increasing trend by the year 2015 (47.7\%) [11]. In a recent review article, Lin et al. reported the wide range of hypercholesterolaemia prevalence in the Asia Pacific region [8]. Comparing with other South East Asian countries, the prevalence of hypercholesterolaemia were lower at $46.9 \%$ in the Philippines and $35.8 \%$ in Indonesia [7, 17]. The prevalence in Singapore, based on its National Health Survey 2010 which included adults 18 years and above, was comparatively lower at $17.4 \%$ [18]. However, this was based on a cut-off of $\geq 6.2 \mathrm{mmol} / \mathrm{L}$. When the cut-off was taken at a similar level to this study $(\geq 5.2 \mathrm{mmol} / \mathrm{L})$, the prevalence was $51.5 \%$.

In our study, the prevalence of elevated LDL-c was found to be high at $56.7 \%$. Nawawi et al. studied 609 rural Malays and found a comparable prevalence of 57.2\% [12]. Another smaller study involving 148 factory workers in Kelantan reported a prevalence of $38.2 \%$ [13]. This lower prevalence may be explained by the higher cut-off used 
Table 4 Mean TG and prevalence of elevated TG according to personal and clinical attributes

\begin{tabular}{|c|c|c|c|c|c|}
\hline & \multirow[t]{2}{*}{ Mean $( \pm S D)$} & \multirow[b]{2}{*}{$p$ value } & \multicolumn{3}{|l|}{ Elevated TG } \\
\hline & & & $\begin{array}{l}\text { No } \\
\text { n (\%) }\end{array}$ & $\begin{array}{l}\text { Yes } \\
\text { n (\%) }\end{array}$ & $p$ value \\
\hline $\begin{array}{l}\text { Age groups (years) } \\
30-39 \\
40-49 \\
50-59 \\
\geq 60\end{array}$ & $\begin{array}{l}1.61( \pm 1.05) \\
1.71( \pm 1.07) \\
1.81( \pm 1.07) \\
1.80( \pm 0.96)\end{array}$ & $<0.001^{* *}$ & $\begin{array}{l}792(70.2) \\
1995(65.4) \\
1874(59.4) \\
1617(59.9)\end{array}$ & $\begin{array}{l}336(29.8) \\
1056(34.6) \\
1279(40.6) \\
1082(40.1)\end{array}$ & $<0.001^{* *}$ \\
\hline $\begin{array}{l}\text { Gender } \\
\text { Male } \\
\text { Female }\end{array}$ & $\begin{array}{l}1.95( \pm 1.14) \\
1.60( \pm 0.93)\end{array}$ & $<0.001^{* *}$ & $\begin{array}{l}2319(53.8) \\
3959(69.2)\end{array}$ & $\begin{array}{l}1992(46.2) \\
1761(30.8)\end{array}$ & $<0.001^{* *}$ \\
\hline $\begin{array}{l}\text { Ethnicity } \\
\text { Malay } \\
\text { Chinese } \\
\text { Indian } \\
\text { Indigenous }\end{array}$ & $\begin{array}{l}1.79( \pm 1.05) \\
1.52( \pm 1.01) \\
1.77( \pm 1.12) \\
1.70( \pm 0.98)\end{array}$ & $<0.001^{* *}$ & $\begin{array}{l}4427(60.7) \\
730(72.1) \\
173(63.6) \\
948(65.5)\end{array}$ & $\begin{array}{l}2871(39.3) \\
283(27.9) \\
99(36.4) \\
500(34.5)\end{array}$ & $<0.001^{* *}$ \\
\hline $\begin{array}{l}\text { Education attainment } \\
\text { No formal education } \\
\text { Primary } \\
\text { Secondary } \\
\text { Tertiary }\end{array}$ & $\begin{array}{l}1.80( \pm 0.99) \\
1.82( \pm 1.02) \\
1.72( \pm 1.01) \\
1.65( \pm 1.12)\end{array}$ & $<0.001^{* *}$ & $\begin{array}{l}873(60.0) \\
1487(59.8) \\
2227(64.0) \\
1147(67.3)\end{array}$ & $\begin{array}{l}581(40.0) \\
1000(40.2) \\
1255(36.0) \\
558(32.7)\end{array}$ & $<0.001^{* *}$ \\
\hline $\begin{array}{l}\text { Location } \\
\text { Urban } \\
\text { Rural }\end{array}$ & $\begin{array}{l}1.68( \pm 1.04) \\
1.82( \pm 1.04)\end{array}$ & $<0.001^{* *}$ & $\begin{array}{l}3298(65.6) \\
2980(59.6)\end{array}$ & $\begin{array}{l}1733(34.5) \\
2020(40.4)\end{array}$ & $<0.001^{* *}$ \\
\hline $\begin{array}{l}\text { Smoking status } \\
\text { Non-smoker } \\
\text { Previous smoker } \\
\text { Current smoker }\end{array}$ & $\begin{array}{l}1.68( \pm 0.98) \\
1.84( \pm 1.05) \\
2.08( \pm 1.22)\end{array}$ & $<0.001^{* *}$ & $\begin{array}{l}4727(65.8) \\
597(57.0) \\
603(49.3)\end{array}$ & $\begin{array}{l}2459(34.2) \\
450(43.0) \\
621(50.7)\end{array}$ & $<0.001^{* *}$ \\
\hline $\begin{array}{l}\text { Diabetes mellitus } \\
\text { No } \\
\text { Yes }\end{array}$ & $\begin{array}{l}1.67( \pm 0.95) \\
2.23( \pm 1.34)\end{array}$ & $<0.001^{* *}$ & $\begin{array}{l}5601(65.9) \\
677(44.2)\end{array}$ & $\begin{array}{l}2898(34.1) \\
855(55.8)\end{array}$ & $<0.001^{* *}$ \\
\hline $\begin{array}{l}\text { Hypertension } \\
\text { No } \\
\text { Yes }\end{array}$ & $\begin{array}{l}1.63( \pm 1.00) \\
1.89( \pm 1.07)\end{array}$ & $<0.001^{* *}$ & $\begin{array}{l}3700(68.8) \\
2578(55.4)\end{array}$ & $\begin{array}{l}1675(31.2) \\
2078(44.6)\end{array}$ & $<0.001^{* *}$ \\
\hline $\begin{array}{l}\text { Body mass index } \\
\text { Underweight } \\
\text { Normal } \\
\text { Overweight } \\
\text { Obese }\end{array}$ & $\begin{array}{l}1.33( \pm 0.70) \\
1.49( \pm 0.87) \\
1.76( \pm 1.02) \\
1.97( \pm 1.13)\end{array}$ & $<0.001^{* *}$ & $\begin{array}{l}307(82.8) \\
1717(75.1) \\
2271(61.9) \\
1681(52.3)\end{array}$ & $\begin{array}{l}64(17.3) \\
570(24.9) \\
1399(38.1) \\
1531(47.7)\end{array}$ & $<0.001^{* *}$ \\
\hline $\begin{array}{l}\text { Waist-hip ratio } \\
\text { Normal } \\
\text { Abdominal obesity }\end{array}$ & $\begin{array}{l}1.50( \pm 0.82) \\
2.00( \pm 1.16)\end{array}$ & $<0.001^{* *}$ & $\begin{array}{l}3302(74.6) \\
2321(51.0)\end{array}$ & $\begin{array}{l}1123(25.4) \\
2232(49.0)\end{array}$ & $<0.001^{* *}$ \\
\hline
\end{tabular}

* significant at $p<0.05 ;{ }^{* *}$ significant at $p<0.001$

$(\geq 4.1 \mathrm{mmol} / \mathrm{L})$. The prevalence of elevated LDL-c in the Philippines was $47.2 \%$ [17] and 15.2\% in Singapore; both reports included adults 18 years and above, and Singapore used a higher cut-off of $\geq 4.1 \mathrm{mmol} / \mathrm{L}$ [18].

This study found the prevalence of elevated TG to be $37.4 \%$, which is slightly lower to that reported by Nawawi et al. (46.1\%) [12] and Nazri et al. (42.1\%) [13]. Prevalence of this dyslipidaemia subtype was found to be similar in the Philippines and Thailand, both at 38.6\% [17, 19]. For low HDL-c, the prevalence was $36.2 \%$ in this study, compared to Nawawi et al. (13.1\%) [12] and Nazri et al. (9.2\%) [13]. These differences could be contributed by the lower cut off $(<0.9 \mathrm{mmol} / \mathrm{L}$ for both males and females) used by Nawawi et al., and the influence of dietary intake and physical activity levels $[12,13]$. Thailand had a prevalence of $47.1 \%$ [19], but the Philippines had a very high of prevalence of $71.3 \%$ [17]. The reasons for this great difference is not apparent but a combination of genetic, nutritional and environmental factors had been proposed [20].

Recent evidence suggests that non-HDL-c is a more powerful CV risk predictor compared to LDL-c [24]. The prevalence of this dyslipidaemia subtype was $56.2 \%$ in our study. There are no previous published local studies on this subtype of dyslipidaemia. A study in Colombia reported a very high prevalence of $75.3 \%$, making it their most prevalent dyslipidaemia subtype 
Table 5 Mean HDL-c and prevalence of low HDL-c according to personal and clinical attributes

\begin{tabular}{|c|c|c|c|c|c|}
\hline & \multirow[t]{2}{*}{ Mean $( \pm S D)$} & \multirow[b]{2}{*}{$p$ value } & \multicolumn{3}{|l|}{ Low HDL-C } \\
\hline & & & $\begin{array}{l}\text { No } \\
\text { n (\%) }\end{array}$ & $\begin{array}{l}\text { Yes } \\
\text { n (\%) }\end{array}$ & $p$ value \\
\hline $\begin{array}{l}\text { Age groups (years) } \\
30-39 \\
40-49 \\
50-59 \\
\geq 60\end{array}$ & $\begin{array}{l}1.36( \pm 0.76) \\
1.28( \pm 0.50) \\
1.27( \pm 0.51) \\
1.28( \pm 0.47)\end{array}$ & $<0.001^{* *}$ & $\begin{array}{l}714(62.9) \\
1920(62.8) \\
1997(63.3) \\
1784(66.1)\end{array}$ & $\begin{array}{l}421(37.1) \\
1137(37.2) \\
1159(36.7) \\
915(33.9)\end{array}$ & $0.041^{*}$ \\
\hline $\begin{array}{l}\text { Gender } \\
\text { Male } \\
\text { Female }\end{array}$ & $\begin{array}{l}1.15( \pm 0.46) \\
1.39( \pm 0.56)\end{array}$ & $<0.001^{* *}$ & $\begin{array}{l}2731(63.3) \\
3684(64.3)\end{array}$ & $\begin{array}{l}1586(36.7) \\
2046(35.7)\end{array}$ & 0.287 \\
\hline $\begin{array}{l}\text { Ethnicity } \\
\text { Malay } \\
\text { Chinese } \\
\text { Indian } \\
\text { Indigenous }\end{array}$ & $\begin{array}{l}1.26( \pm 0.42) \\
1.50( \pm 0.65) \\
1.17( \pm 0.35) \\
1.26( \pm 0.86)\end{array}$ & $<0.001^{* *}$ & $\begin{array}{l}4743(64.9) \\
820(81.0) \\
157(57.7) \\
695(48.0)\end{array}$ & $\begin{array}{l}2571(35.2) \\
193(19.1) \\
115(42.3) \\
753(52.0)\end{array}$ & $<0.001^{* *}$ \\
\hline $\begin{array}{l}\text { Education attainment } \\
\text { No formal education } \\
\text { Primary } \\
\text { Secondary } \\
\text { Tertiary }\end{array}$ & $\begin{array}{l}1.25( \pm 0.60) \\
1.26( \pm 0.50) \\
1.29( \pm 0.53) \\
1.31( \pm 0.47)\end{array}$ & $0.004^{*}$ & $\begin{array}{l}806(55.4) \\
1558(62.7) \\
2234(64.1) \\
1200(70.3)\end{array}$ & $\begin{array}{l}648(44.6) \\
929(37.4) \\
1249(35.9) \\
506(29.7)\end{array}$ & $<0.001^{* *}$ \\
\hline $\begin{array}{l}\text { Location } \\
\text { Urban } \\
\text { Rural }\end{array}$ & $\begin{array}{l}1.29( \pm 0.38) \\
1.28( \pm 0.65)\end{array}$ & 0.964 & $\begin{array}{l}3407(67.7) \\
3008(60.0)\end{array}$ & $\begin{array}{l}1627(32.3) \\
2005(40.0)\end{array}$ & $<0.001^{* *}$ \\
\hline $\begin{array}{l}\text { Smoking status } \\
\text { Non-smoker } \\
\text { Previous smoker } \\
\text { Current smoker }\end{array}$ & $\begin{array}{l}1.32( \pm 0.52) \\
1.19( \pm 0.47) \\
1.08( \pm 0.37)\end{array}$ & $<0.001^{* *}$ & $\begin{array}{l}4680(65.1) \\
667(63.7) \\
665(54.3)\end{array}$ & $\begin{array}{l}2508(34.9) \\
380(36.3) \\
560(45.7)\end{array}$ & $<0.001^{* *}$ \\
\hline $\begin{array}{l}\text { Diabetes mellitus } \\
\text { No } \\
\text { Yes }\end{array}$ & $\begin{array}{l}1.30( \pm 0.54) \\
1.19( \pm 0.45)\end{array}$ & $<0.001^{* *}$ & $\begin{array}{l}5557(65.3) \\
858(55.9)\end{array}$ & $\begin{array}{l}2956(34.7) \\
676(44.1)\end{array}$ & $<0.001^{* *}$ \\
\hline $\begin{array}{l}\text { Hypertension } \\
\text { No } \\
\text { Yes }\end{array}$ & $\begin{array}{l}1.30( \pm 0.52) \\
1.27( \pm 0.54)\end{array}$ & $<0.001^{* *}$ & $\begin{array}{l}3501(64.9) \\
2914(62.6)\end{array}$ & $\begin{array}{l}1890(35.1) \\
1742(37.4)\end{array}$ & $0.014^{*}$ \\
\hline $\begin{array}{l}\text { Body mass index } \\
\text { Underweight } \\
\text { Normal } \\
\text { Overweight } \\
\text { Obese }\end{array}$ & $\begin{array}{l}1.46( \pm 0.63) \\
1.40( \pm 0.64) \\
1.27( \pm 0.50) \\
1.19( \pm 0.44)\end{array}$ & $<0.001^{* *}$ & $\begin{array}{l}269(72.5) \\
1700(74.3) \\
2375(64.7) \\
1749(54.5)\end{array}$ & $\begin{array}{l}102(27.5) \\
588(25.7) \\
1297(35.3) \\
1463(45.6)\end{array}$ & $<0.001^{* *}$ \\
\hline $\begin{array}{l}\text { Waist-hip ratio } \\
\text { Normal } \\
\text { Abdominal obesity }\end{array}$ & $\begin{array}{l}1.39( \pm 0.57) \\
1.19( \pm 0.47)\end{array}$ & $<0.001^{* *}$ & $\begin{array}{l}3177(71.8) \\
2559(56.2)\end{array}$ & $\begin{array}{l}1250(28.2) \\
1995(43.8)\end{array}$ & $<0.001^{* *}$ \\
\hline
\end{tabular}

* significant at $p<0.05 ;{ }^{* *}$ significant at $p<0.001$

[21]. The authors proposed that this is due to the high refined carbohydrate intake in their population. The latest Malaysian dyslipidaemia clinical practice guidelines recommended that non-HDL-c should only be a secondary target, after the LDL-c target has been achieved for patients with combined hyperlipidaemias, diabetes, cardiometabolic risk, or chronic kidney disease [1]. Given the high prevalence rate of this highly atherogenic dyslipidaemia subtype, this recommendation may need to be revised.

Malays, who make up $51 \%$ of the population in this country [22] had the highest prevalence for elevated TC (70.2\%), elevated LDL-c (63.1\%), and elevated
non-HDL-c (62.5\%). The multiple logistic regression model showed that the other ethnicities had lower adjusted odds ratio for these three dyslipidaemia subtypes. Nawawi et al. found the prevalence of these dyslipidaemia subtypes in rural Malays were $67.3 \%$ (high TC) and 57.2\% (high LDL-c) [12]. The 2011 National Health and Morbidity Survey also reported that Malays had the highest prevalence of high TC (38.4\%) [10]. The prevalence rate might be lower because it included adults 18 years old and older. Underlying genetic predisposition, dietary habits and physical activity levels have been proposed as possible causes for the high 
Table 6 Mean non-HDL-c and prevalence of elevated non-HDL-c according to personal and clinical attributes

\begin{tabular}{|c|c|c|c|c|c|}
\hline & \multirow[t]{2}{*}{ Mean $( \pm S D)$} & \multirow[b]{2}{*}{$p$ value } & \multicolumn{3}{|c|}{ Elevated non-HDL-c } \\
\hline & & & $\begin{array}{l}\text { No } \\
\text { n (\%) }\end{array}$ & $\begin{array}{l}\text { Yes } \\
\text { n (\%) }\end{array}$ & $p$ value \\
\hline $\begin{array}{l}\text { Age groups (years) } \\
30-39 \\
40-49 \\
50-59 \\
\geq 60\end{array}$ & $\begin{array}{l}4.03( \pm 1.52) \\
4.33( \pm 1.28) \\
4.58( \pm 1.38) \\
4.48( \pm 1.33)\end{array}$ & $<0.001^{* *}$ & $\begin{array}{l}607(53.5) \\
1410(46.1) \\
1231(39.0) \\
1153(42.7)\end{array}$ & $\begin{array}{l}528(46.5) \\
1647(53.9) \\
1925(61.0) \\
1546(57.3)\end{array}$ & $0.000^{*}$ \\
\hline $\begin{array}{l}\text { Gender } \\
\text { Male } \\
\text { Female }\end{array}$ & $\begin{array}{l}4.53( \pm 1.33) \\
4.33( \pm 1.38)\end{array}$ & $<0.001^{* *}$ & $\begin{array}{l}1693(39.2) \\
2708(47.3)\end{array}$ & $\begin{array}{l}2624(60.8) \\
3022(52.7)\end{array}$ & $<0.001^{* *}$ \\
\hline $\begin{array}{l}\text { Ethnicity } \\
\text { Malay } \\
\text { Chinese } \\
\text { Indian } \\
\text { Indigenous }\end{array}$ & $\begin{array}{l}4.62( \pm 1.27) \\
4.04( \pm 1.31) \\
4.34( \pm 1.15) \\
3.65( \pm 1.52)\end{array}$ & $<0.001^{* *}$ & $\begin{array}{l}2740(37.5) \\
566(55.9) \\
128(47.1) \\
967(66.8)\end{array}$ & $\begin{array}{l}4574(62.5) \\
447(44.1) \\
144(52.9) \\
481(33.2)\end{array}$ & $<0.001^{* *}$ \\
\hline $\begin{array}{l}\text { Education attainment } \\
\text { No formal education } \\
\text { Primary } \\
\text { Secondary } \\
\text { Tertiary }\end{array}$ & $\begin{array}{l}4.26( \pm 1.44) \\
4.51( \pm 1.35) \\
4.46( \pm 1.33) \\
4.41( \pm 1.22)\end{array}$ & $<0.001^{* *}$ & $\begin{array}{l}724(49.8) \\
1006(40.5) \\
1492(42.8) \\
746(43.7)\end{array}$ & $\begin{array}{l}730(50.2) \\
1481(59.6) \\
1991(57.2) \\
960(56.3)\end{array}$ & $<0.001^{* *}$ \\
\hline $\begin{array}{l}\text { Location } \\
\text { Urban } \\
\text { Rural }\end{array}$ & $\begin{array}{l}4.53( \pm 1.19) \\
4.30( \pm 1.51)\end{array}$ & $<0.001^{* *}$ & $\begin{array}{l}2088(41.5) \\
2313(46.1)\end{array}$ & $\begin{array}{l}2946(58.5) \\
2700(53.9)\end{array}$ & $0.002^{*}$ \\
\hline $\begin{array}{l}\text { Smoking status } \\
\text { Non-smoker } \\
\text { Previous smoker } \\
\text { Current smoker }\end{array}$ & $\begin{array}{l}4.39( \pm 1.32) \\
4.51( \pm 1.36) \\
4.63( \pm 1.30)\end{array}$ & $<0.001^{* *}$ & $\begin{array}{l}3246(45.2) \\
418(39.9) \\
457(37.3)\end{array}$ & $\begin{array}{l}3942(54.8) \\
629(60.1) \\
768(62.7)\end{array}$ & $<0.001^{* *}$ \\
\hline $\begin{array}{l}\text { Diabetes mellitus } \\
\text { No } \\
\text { Yes }\end{array}$ & $\begin{array}{l}4.37( \pm 1.35) \\
4.67( \pm 1.37)\end{array}$ & $<0.001^{* *}$ & $\begin{array}{l}3857(45.3) \\
544(35.5)\end{array}$ & $\begin{array}{l}4656(54.7) \\
990(64.5)\end{array}$ & $<0.001^{* *}$ \\
\hline $\begin{array}{l}\text { Hypertension } \\
\text { No } \\
\text { Yes }\end{array}$ & $\begin{array}{l}4.30( \pm 1.33) \\
4.55( \pm 1.38)\end{array}$ & $<0.001^{* *}$ & $\begin{array}{l}2598(48.2) \\
1803(38.7)\end{array}$ & $\begin{array}{l}2793(51.8) \\
2853(61.3)\end{array}$ & $<0.001^{* *}$ \\
\hline $\begin{array}{l}\text { Body mass index } \\
\text { Underweight } \\
\text { Normal } \\
\text { Overweight } \\
\text { Obese }\end{array}$ & $\begin{array}{l}3.73( \pm 1.21) \\
4.06( \pm 1.37) \\
4.52( \pm 1.33) \\
4.67( \pm 1.27)\end{array}$ & $<0.001^{* *}$ & $\begin{array}{l}260(70.1) \\
1301(56.9) \\
1482(40.4) \\
1105(34.4)\end{array}$ & $\begin{array}{l}111(29.9) \\
987(43.1) \\
2190(59.6) \\
2107(65.6)\end{array}$ & $<0.001^{* *}$ \\
\hline $\begin{array}{l}\text { Waist-hip ratio } \\
\text { Normal } \\
\text { Abdominal obesity }\end{array}$ & $\begin{array}{l}4.21( \pm 1.31) \\
4.64( \pm 1.34)\end{array}$ & $<0.001^{* *}$ & $\begin{array}{l}2261(51.1) \\
1659936.4)\end{array}$ & $\begin{array}{l}2166(48.9) \\
2895(63.6)\end{array}$ & $<0.001^{* *}$ \\
\hline
\end{tabular}

* significant at $p<0.05 ;{ }^{* *}$ significant at $p<0.001$

prevalence of these dyslipidaemia subtypes in Malays [23].

Interestingly, the Indigenous population was associated with almost 2.5 times increased odds ratio to have low HDL-c [aOR 2.42 (95\% CI 2.08-2.81)], compared to Malays. This is a heterogenous group composed of multiple ethnic groups, with the majority from the Kadazan-Dusun ethnic from East Malaysia. There is no previous published data for comparison. Further studies in this population are needed to confirm this observation and determine its significance especially on the occurrence of atherosclerotic cardiovascular disease.
Two measures to characterize body composition were included in this study, which were BMI and WHR. Elevated levels in each method were associated with higher odds ratio of elevated $\mathrm{TC}$ and all dyslipidaemia subtypes. This finding is consistent with previous studies [21]. Although both measures had significant association with increased likelihood of elevated TC and all dyslipidaemia subtypes, the adjusted OR for BMI were higher compared to WHR. This suggest that BMI has a stronger association with dyslipidaemia in our population. 
Table 7 Personal and clinical attributes associated with subtypes of dyslipidaemia

\begin{tabular}{|c|c|c|c|c|c|}
\hline & $\begin{array}{l}\text { High TC } \\
\text { aOR (95\% Cl) }\end{array}$ & $\begin{array}{l}\text { High LDL-c } \\
\text { aOR }(95 \% \mathrm{Cl})\end{array}$ & $\begin{array}{l}\text { High TG } \\
\text { aOR (95\% Cl) }\end{array}$ & $\begin{array}{l}\text { Low HDL-c } \\
\text { aOR (95\% Cl) }\end{array}$ & $\begin{array}{l}\text { High Non-HDL-c } \\
\text { aOR }(95 \% \mathrm{Cl})\end{array}$ \\
\hline Age (years) & 1.00 & 1.00 & 1.00 & 1.00 & 1.00 \\
\hline $30-39$ & $1.28(1.09-1.50)$ & $1.25(1.07-1.46)$ & $1.05(0.88-1.25)$ & $0.93(0.78-1.09)$ & $1.21(1.03-1.42)$ \\
\hline $40-49$ & $1.78(1.50-2.10)$ & $1.54(1.31-1.82)$ & $1.15(0.96-1.38)$ & $0.81(0.68-0.96)$ & $1.50(1.27-1.77)$ \\
\hline $\begin{array}{l}50-59 \\
\geq 60\end{array}$ & $1.58(1.31-1.90)$ & $1.42(1.18-1.71)$ & $1.00(0.82-1.21)$ & $0.67(0.55-0.81)$ & $1.30(1.08-1.57)$ \\
\hline Gender & 1.00 & 1.00 & 1.00 & 1.00 & 1.00 \\
\hline $\begin{array}{l}\text { Male } \\
\text { Female }\end{array}$ & $1.12(1.00-1.26)$ & $0.98(0.87-1.09)$ & $0.62(0.55-0.70)$ & $1.14(1.02-1.29)$ & $0.79(0.71-0.88)$ \\
\hline Ethnicity & 1.00 & 1.00 & 1.00 & 1.00 & 1.00 \\
\hline Malay & $0.70(0.60-0.83)$ & $0.58(0.49-0.68)$ & $0.92(0.77-1.11)$ & $0.55(0.45-0.67)$ & $0.60(0.51-0.71)$ \\
\hline Chinese & $0.60(0.45-0.80)$ & $0.61(0.46-0.81)$ & $0.90(0.66-1.23)$ & $1.28(0.95-1.72)$ & $0.63(0.47-0.84)$ \\
\hline $\begin{array}{l}\text { Indian } \\
\text { Indigenous }\end{array}$ & $0.27(0.23-0.32)$ & $0.29(0.25-0.34)$ & $0.88(0.75-1.03)$ & $2.42(2.08-2.81)$ & $0.33(0.28-0.39)$ \\
\hline Educational attainment & 1.00 & 1.00 & 1.00 & 1.00 & 1.00 \\
\hline No formal & $1.06(0.91-1.24)$ & $1.04(0.90-1.21)$ & $0.78(0.67-0.91)$ & $0.76(0.65-0.88)$ & $0.98(0.84-1.14)$ \\
\hline Primary & $1.07(0.91-1.26)$ & $1.02(0.87-1.20)$ & $0.76(0.65-0.90)$ & $0.71(0.61-0.84)$ & $0.88(0.75-1.04)$ \\
\hline $\begin{array}{l}\text { Secondary } \\
\text { Tertiary }\end{array}$ & $1.04(0.85-1.27)$ & $0.99(0.81-1.20)$ & $0.75(0.62-0.92)$ & $0.60(0.49-0.73)$ & $0.86(0.71-1.05)$ \\
\hline Location & 1.00 & 1.00 & 1.00 & 1.00 & 1.00 \\
\hline $\begin{array}{l}\text { Urban } \\
\text { Rural }\end{array}$ & $0.95(0.85-1.07)$ & $0.84(0.75-0.95)$ & $1.39(1.24-1.57)$ & $0.99(0.88-1.11)$ & $1.01(0.90-1.13)$ \\
\hline Smoking & 1.00 & 1.00 & 1.00 & 1.00 & 1.00 \\
\hline Non-smoker & $1.03(0.87-1.22)$ & $1.00(0.85-1.17)$ & $1.04(0.88-1.22)$ & $1.11(0.95-1.31)$ & $1.01(0.86-1.19)$ \\
\hline $\begin{array}{l}\text { Previous smoker } \\
\text { Current smoker }\end{array}$ & $1.06(0.91-1.25)$ & $1.14(0.98-1.33)$ & $1.49(1.27-1.74)$ & $1.76(1.50-2.06)$ & $1.25(1.06-1.46)$ \\
\hline Body mass index & 1.00 & 1.00 & 1.00 & 1.00 & 1.00 \\
\hline Normal & $0.61(0.48-0.78)$ & $0.55(0.43-0.71)$ & $0.72(0.53-0.98)$ & $1.04(0.79-1.37)$ & $0.53(0.41-0.69)$ \\
\hline Underweight & $1.36(1.20-1.54)$ & $1.45(1.29-1.64)$ & $1.67(1.46-1.91)$ & $1.65(1.44-1.89)$ & $1.66(1.47-1.87)$ \\
\hline $\begin{array}{l}\text { Overweight } \\
\text { Obese }\end{array}$ & $1.36(1.19-1.56)$ & $1.43(1.26-1.63)$ & $2.34(2.03-2.70)$ & $2.51(2.17-2.89)$ & $1.91(1.67-2.18)$ \\
\hline Waist-hip ratio & 1.00 & 1.00 & 1.00 & 1.00 & 1.00 \\
\hline $\begin{array}{l}\text { Normal } \\
\text { Abdominal obesity }\end{array}$ & $1.17(1.05-1.30)$ & $1.12(1.01-1.23)$ & $1.86(1.67-2.06)$ & $1.62(1.46-1.80)$ & $1.33(1.21-1.48)$ \\
\hline Diabetes mellitus & 1.00 & 1.00 & 1.00 & 1.00 & 1.00 \\
\hline $\begin{array}{l}\text { No } \\
\text { Yes }\end{array}$ & $0.89(0.78-1.02)$ & $0.78(0.69-0.89)$ & $1.90(1.67-2.16)$ & $1.34(1.18-1.53)$ & $1.06(0.93-1.21)$ \\
\hline Hypertension & 1.00 & 1.00 & 1.00 & 1.00 & 1.00 \\
\hline $\mathrm{No}$ & $1.16(1.04-1.28)$ & $1.10(1.00-1.21)$ & $1.36(1.23-1.51)$ & $0.95(0.85-1.05)$ & $1.20(1.09-1.33)$ \\
\hline
\end{tabular}

Multivariable regression model, controlled for age, gender, ethnicity, educational attainment, locality, smoking status, BMI, waist-hip ratio, diabetes mellitus status, hypertension status. No significant interactions

\section{Strengths and limitations of the study}

The strengths of this study include the large sample size and high response rate, strengthening the external validity of the findings. However, as a community activity where participation was on a voluntary basis, all eligible participants who attended were recruited, resulting in the total number of participants recruited exceeding the calculated sample size. Given the voluntary nature of participating in this study, there is a possibility that the participants were more health-conscious than the general population. Malays were over-represented in this study while Chinese and Indians were under-represented. Our study used the Malaysian guidelines as the cut-off points for dyslipidaemia. Therefore, this might affect direct comparisons with studies utilizing other guidelines. Finally, this study looked at the cross-sectional baseline data hence the findings can only show association but not causality. Therefore, interpretation and generalization of the results should be done with care.

\section{Implications for clinical practice and future research}

REDISCOVER provided important insights on the current magnitude and associated factors of dyslipidaemia among Malaysians. Knowing the high prevalence of these dyslipidaemia subtypes may raise the clinicians' awareness of this issue and ensure a holistic approach to managing 
dyslipidaemia. Laboratory reporting of non-HDL-c as part of the lipid profile should also be introduced to raise awareness of its importance among clinicians. Given the high prevalence of dyslipidaemia, immediate actions are needed now. These should include opportunistic screening for all 30 years old and older, Malays, those who are overweight or centrally obese, and those with hypertension or diabetes. As obesity has been consistently shown to be associated with CVD and its risk factors, more effective public health measures need to be implemented to educate all Malaysians regarding the importance of healthy and balanced dietary intake, and adequate physical activity levels to address this epidemic. Further studies involving dietary intake and physical activity among Malaysians, as well as looking into the association between the Indigenous population with low HDL-c levels may provide valuable information on dyslipidaemia in this country. More research to establish the relationship between elevated non-HDL-c and atherosclerotic CVD in Malaysians is also needed. All these will form the evidence-base for future local dyslipidaemia management guidelines and practice.

\section{Conclusions}

The REDISCOVER Study found that the prevalence of elevated TC and all dyslipidaemia subtypes is worryingly high in Malaysian adults, where increased body mass seems to be the main driver. Differences of the dyslipidaemia subtype prevalence between personal and clinical attributes of individuals observed may have specific use in the realm of precision medicine such that a more targeted approach may be employed in the prevention and treatment of dyslipidaemia.

\section{Abbreviations}

aOR: Adjusted odds ratio; BMI: Body mass index; BP: Blood pressure; Cl: Confidence interval; CVD: Cardiovascular diseases; HDL-c: High-density lipoprotein cholesterol; LDL-c: Low-density lipoprotein cholesterol; NHMS: National Health and Morbidity Survey; Non-HDL-c: Non-high-density lipoprotein cholesterol; OR: Odds ratio; SD: Standard deviation; TC: Total cholesterol; TG: Triglycerides; VLDL: Very low-density lipoprotein; WHR: Waist-hip ratio.

\section{Acknowledgements}

The authors would like to thank all REDISCOVER investigators and the participants of the study.

\footnotetext{
Authors' contributions

MSMY wrote the manuscript and was involved in the data collection of the REDISCOVER Study. AMD and MM performed the statistical analysis and contributed to the critical discussion of the results. NB, AMD, NAB, ASR, SAR, and $\mathrm{N}$-AMNK contributed to the critical revision of the manuscript and were involved in the data collection. KY is the Principal Investigator of this study. He played a major role in the conception and design of the study, contributed to the critical revision of the manuscript and intellectual content, and provided expertise and oversight throughout the process. All authors read and approved the final version. All authors agreed to be personally accountable for the author's own contributions and to ensure that questions related to the accuracy or integrity of any part of the work, even ones in which the author was not personally involved, are appropriately investigated, resolved, and the resolution documented in the literature.
}

\section{Funding}

This research is funded by the Ministry of Higher Education, Malaysia (Grant no. 600-RMI/LRGS 5/3 (2/2011)) and Ministry of Science, Technology and Innovation, Malaysia (Grant no. 07-05-IFN BPH 010). The funding bodies did not have a role in the design of the study and collection, analysis, and interpretation of data and in writing the manuscript.

\section{Availability of data and materials}

Data are kept at the Centre for Translational Research and Epidemiology (CenTRE), Faculty of Medicine, Universiti Teknologi MARA. Data will be shared upon request and it is subjected to the data protection regulation.

\section{Declarations}

\section{Ethics approval and consent to participate}

This study was approved by the Research Ethics Committee of Universiti Teknologi MARA. Reference number: REC/UITM/2007(10). Written informed consent was obtained from all participants prior to their study enrolment.

\section{Consent for publication \\ Not applicable.}

\section{Competing interests}

The authors declare no competing interests.

\section{Author details}

${ }^{1}$ Department of Primary Care Medicine, Faculty of Medicine, Universiti Teknologi MARA, Selayang Campus, Jalan Prima Selayang 7, 68100 Batu Caves, Selangor, Malaysia. ${ }^{2}$ Department of Community Medicine, School of Medicine, International Medical University, Bukit Jalil, 57000 Kuala Lumpur, Malaysia. ${ }^{3}$ Centre for Translational Research and Epidemiology (CenTRE), Faculty of Medicine, Universiti Teknologi MARA, Jalan Hospital, 47000 Sungai Buloh, Selangor, Malaysia. ${ }^{4}$ Institute of Pathology, Laboratory and Forensic Medicine (I-PPerForM), Universiti Teknologi MARA, Sungai Buloh Campus, Jalan Hospital, 47000 Sungai Buloh, Selangor, Malaysia. ${ }^{5}$ Department of Physiology, Faculty of Medicine, Universiti Teknologi MARA, Sungai Buloh Campus, Jalan Hospital, 47000 Sungai Buloh, Selangor, Malaysia. ${ }^{6}$ Department of Public Health Medicine, Faculty of Medicine, Universiti Teknologi MARA, Sungai Buloh Campus, Jalan Hospital, 47000 Sungai Buloh, Selangor, Malaysia. ${ }^{7}$ Faculty of Medicine and Health Sciences, UCSI University, No.1, Jalan Menara Gading, UCSI Heights, Taman Connaught, 56000 Cheras, Kuala Lumpur, Malaysia. ${ }^{8}$ Faculty of Medicine, Universiti Teknologi MARA, Sungai Buloh Campus, Jalan Hospital, 47000 Sungai Buloh, Selangor, Malaysia.

Received: 9 June 2020 Accepted: 15 March 2021

Published online: 23 March 2021

\section{References}

1. Health Technology Assessment Unit. Clinical Practice Guidelines on Management of Dyslipidaemia: Ministry of Health Malaysia; 2017 [cited 2020 9 January].

2. Chapman MJ, Ginsberg HN, Amarenco P, Andreotti F, Boren J, Catapano $\mathrm{AL}$, et al. Triglyceride-rich lipoproteins and high-density lipoprotein cholesterol in patients at high risk of cardiovascular disease: evidence and guidance for management. Eur Heart J. 2011;32(11):1345-61.

3. Emerging Risk Factors Collab., Di Angelantonio E, Sarwar N, Perry P, Kaptoge S, Ray KK, et al. Major lipids, apolipoproteins, and risk of vascular disease. JAMA. 2009;302(18):1993-2000.

4. Robinson JG, Wang S, Smith BJ, Jacobson TA. Meta-analysis of the relationship between non-high-density lipoprotein cholesterol reduction and coronary heart disease risk. J Am Coll Cardiol. 2009;53(4):316-22.

5. Fruchart JC, Sacks FM, Hermans MP, Assmann G, Brown WV, Ceska R, et al. The Residual Risk Reduction Initiative: a call to action to reduce residual vascular risk in dyslipidaemic patient. Diab Vasc Dis Res. 2008;5(4):319-35.

6. Reiner Ž. Hypertriglyceridaemia and risk of coronary artery disease. Nat Rev Cardiol. 2017;14(7):401-11. 
7. World Health Organization. Global health observatory data repository Geneva: World Health Organization; 2016 [cited 20204 January]. Available from: https://apps.who.int/gho/data/view.main.2467?lang=en.

8. Lin CF, Chang YH, Chien SC, Lin YH, Yeh HY. Epidemiology of Dyslipidemia in the Asia Pacific Region. Int J Gerontol. 2018;12(1):2-6.

9. Institute for Public Health. National Health and Morbidity Survey III: Ministry of Health Malaysia; 2006 [cited 20207 January]. Available from: http:// www.iku.gov.my/index.php/statistics/summary-of-nhms-report-on-disea se-prevalence.

10. Institute for Public Health. National Health and Morbidity Survey IV: Ministry of Health Malaysia; 2011 [cited 20207 January]. Available from: http:// www.iku.gov.my/index.php/statistics/summary-of-nhms-report-on-disea se-prevalence.

11. Institute for Public Health. National Health and Morbidity Survey V: Ministry of Health Malaysia; 2015 [cited 20206 January]. Available from: http:// www.iku.gov.my/index.php/statistics/summary-of-nhms-report-on-disea se-prevalence.

12. Nawawi HM, Nor IM, Noor IM, Karim NA, Arshad F, Khan R, et al. Current status of coronary risk factors among rural Malays in Malaysia. J Cardiovasc Risk. 2002;9(1):17-23.

13. Nazri SM, Tengku MA, Winn T. Lipid disorders among male factory shift workers in Kota Bharu. Kelantan Med J Malaysia. 2007;62(2):134-8.

14. Department of Statistics Malaysia. Population distribution and basic demographic characteristics 2000: Department of Statistics Malaysia; 2000 [cited 20208 January]. Available from: https://www.dosm.gov. my/v1/index.php?r=column/cthemeByCat\&cat=117\&bul_id=MDMxd HZjWTk1SjFzTzNkRXYzcVZjdz09\&menu_id=L0pheU43NWJwRWVSZkIW dzQ4TIhUUT09.

15. Health Technology Assessment Section. Clinical Practice Guidelines on Management of Obesity. Putrajaya: Ministry of Health Malaysia; 2004 [cited 20209 January].
16. World Health Organization. Waist circumference and waist-hip ratio report of a WHO expert consultation. Geneva: World Health Organization; 2008.

17. Food and Nutrition Research Institute. Annual report 2014. Taguig City: Department of Science and Technology; 2014.

18. Epidemiology \& Disease Control Division. National Health Survey 2010. Singapore: Ministry of Health Singapore; 2010.

19. Aekplakorn W, Taneepanichskul S, Kessomboon P, Chongsuvivatwong V, Putwatana P, Sritara P, et al. Prevalence of Dyslipidemia and Management in the Thai Population, National Health Examination Survey IV, 2009. J Lipids. 2014;2014:249584.

20. Rutherford JN, McDade TW, Feranil AB, Adair LS, Kuzawa CW. High prevalence of low HDL-c in the Philippines compared to the US: population differences in associations with diet and BMI. Asia Pac J Clin Nutr. 2010;19(1):57-67.

21. Camacho PA, Otero J, Perez M, Arcos E, Garcia H, Narvaez C, et al. The spectrum of the dyslipidemia in Colombia: the PURE study. Int J Cardiol. 2019;284:111-7.

22. Department of Information Malaysia. Population by states and ethnic group 2015 Kuala Lumpur2016 [cited 2020 10th May]. Available from: https://web.archive.org/web/20160215104349/http://pmr.penerangan. gov.my/index.php/info-terkini/19463-unjuran-populasi-penduduk-2015. html.

23. Al-Khateeb A, Mohamed MS, Imran K, Ibrahim S, Zilfalil BA, Yusof Z. Lipid profile parameters in Malaysian dyslipidemic patients. Kobe J Med Sci. 2011;57(2):E38-48.

\section{Publisher's Note}

Springer Nature remains neutral with regard to jurisdictional claims in published maps and institutional affiliations.
Ready to submit your research? Choose BMC and benefit from:

- fast, convenient online submission

- thorough peer review by experienced researchers in your field

- rapid publication on acceptance

- support for research data, including large and complex data types

- gold Open Access which fosters wider collaboration and increased citations

- maximum visibility for your research: over 100M website views per year

At BMC, research is always in progress.

Learn more biomedcentral.com/submissions 